\title{
PROBING THE PRECESSION OF THE INNER ACCRETION DISK IN CYGNUS X-1
}

\author{
Diego F. Torres, ${ }^{1}$ Gustavo E. Romero, ${ }^{2}$ Xavier Barcons, ${ }^{3}$ and Youjun Lu ${ }^{4}$ \\ Received 2004 December 8; accepted 2005 March 7
}

\begin{abstract}
We show that changes in the orientation of the inner accretion disk of Cyg X-1 affect the shape of the broad Fe K $\alpha$ emission line emitted from this object, in such a way that eV-level spectral resolution observations (such as those that will be carried out by the Astro-E2 satellite) can be used to analyze the dynamics of the disk. We present here a potential diagnostic tool supported by numerical simulations by which a few observations of Cyg X-1, separated in time, can determine whether its accretion disk actually precesses, and if so, determine its period and precession angle. This approach could also be used for similar studies in other microquasar systems.
\end{abstract}

Subject headings: X-rays: binaries - X-rays: individual (Cygnus X-1)

Online material: color figures

\section{INTRODUCTION}

Cyg $\mathrm{X}-1$ is a 5.6 day X-ray binary that harbors the beststudied black hole candidate in the Galaxy. The mass of the accreting compact object has been estimated as $\sim 10.1 M_{\odot}$, and the donor star is classified as an O9.7 Iab supergiant of $\sim 17.8 M_{\odot}$ (Herrero et al. 1995). The system is located at $\sim 2 \mathrm{kpc}$ (e.g., Gierliński et al. 1999). The black hole accretes through the wind of the companion star. Most of the time, the X-ray source is in the so-called low/hard state, characterized by a relatively weak blackbody component peaking at a few $\mathrm{keV}$ plus a strong, hard power law of photon index $\sim 1.6$. A nonthermal radio jet has been observed in this state (Stirling et al. 2001), extending up to $\sim 15$ mas. The jet seems to form an average angle with the line of sight of $\sim 30^{\circ}$ (Fender 2001), and it has been suggested that it might be precessing (Stirling et al. 2001; Romero et al. 2002). Occasionally, a transition to a high/soft state can occur. In this state, most of the radiated energy is concentrated in the blackbody, while the power-law component becomes softer, with an index of $\sim 2.8$, and no jet has been observed.

The usual interpretation of the X-ray behavior of the source is that the blackbody component originates in a cold, optically thick accretion disk, whereas the power-law component is produced in an optically thin hot corona by thermal Comptonization of disk photons (Poutanen et al. 1997; Dove et al. 1997; Esin et al. 1997, 1998). The hot corona fills the inner few tens of gravitational radii around the compact object, while the accretion disk would penetrate only marginally into the coronal region. In the low/hard state the thermal X-ray luminosity is dominated by the corona, with typical luminosities of approximately a few times $10^{37} \mathrm{ergs} \mathrm{s}^{-1}$. During the transition to the high/soft state, the corona is likely ejected as the accretion disk approaches to the black hole (Fender et al. 2004; see also Esin et al. 1997, 1998). Most of the energy is then dissipated by the disk, until the inner part of it dominates the radiation again and the cycle starts again.

\footnotetext{
${ }^{1}$ Lawrence Livermore National Laboratory, 7000 East Avenue, L-413, Livermore, CA 94550; dtorres@igpp.ucllnl.org.

2 Instituto Argentino de Radioastronomía (IAR), C.C. 5, 1894 Villa Elisa, Argentina; romero@irma.iar.unlp.edu.ar.

${ }^{3}$ Instituto de Física de Cantabria (CSIC-UC), 39005 Santander, Spain; barcons@ifca.unican.es.

${ }^{4}$ Center for Astrophysics, University of Science and Technology of China, Hefei, Anhui 230026, China; lyj@astron.berkeley.edu.
}

In the low/hard state, the disk is illuminated by hard photons from the corona resulting in the production of an $\mathrm{Fe} \mathrm{K} \alpha$ line and a Compton reflection feature. The first detection of the line was made by Barr et al. (1985) with EXOSAT (European $X$-Ray Observatory Satellite). They reported a broad (FWHM $\sim 1.2 \mathrm{keV}$ ) emission line at $\sim 6.2 \mathrm{keV}$ with an equivalent width of $\sim 120$ eV. Kitamoto et al. (1990) obtained a Tenma GSPC spectrum that was consistent with a narrow emission line at $\sim 6.5 \mathrm{keV}$ with an equivalent width of $60-80 \mathrm{eV}$. A Compton reflection feature was then found above $20 \mathrm{keV}$ (see Tanaka 1991 and references therein). The Ginga spectrum in the $2-30 \mathrm{keV}$ range can be fitted quite well by the sum of a power law with index $\sim 1.7$, a reflection component, and a narrow $\mathrm{Fe}$ emission line at $6.4 \mathrm{keV}$ with an equivalent width of $\sim 60 \mathrm{eV}$ (Tanaka 1991). Subsequent $A S C A$ observations confirmed these results but restricted the width to $10-30 \mathrm{eV}$ (Ebisawa et al. 1996). A broad edge at $E>7 \mathrm{keV}$ was also reported. A detailed historical account of attempts to detect an Fe line in Cyg X-1 and the intrinsic difficulties it entails can be found in Reynolds \& Nowak (2003). Recently, Chandra observed Cyg X-1 with the High Energy Transmission Grating Spectrometer in an intermediate X-ray state (Miller et al. 2002). The narrow Fe line was detected at $E=$ $6.415 \pm 0.007 \mathrm{keV}$ with an equivalent width of $W=16_{-2}^{+3} \mathrm{eV}$, along with a broad line at $E=5.82 \pm 0.07 \mathrm{keV}$ with $W=$ $140_{-40}^{+70} \mathrm{eV}$. A smeared edge was also detected at $7.3 \pm 0.2 \mathrm{keV}$. Miller et al. (2002) interpret these results in terms of an accretion disk with irradiation of the inner disk producing the broad $\mathrm{Fe} \mathrm{K} \alpha$ emission line and edge, and irradiation of the outer disk producing the narrow line. The broad line is shaped by Doppler and gravitational effects and, to a lesser extent, by Compton reflection.

For different spectral states, the different disk structure may change the $\mathrm{Fe} \mathrm{K} \alpha$ line. For example, if the disk is truncated at a much larger radius rather than the innermost stable orbit, as suggested for the hard state, then the width of the $\mathrm{Fe} \mathrm{K} \alpha$ line may significantly decrease. In any case, the variation due to disk precession, if that were observable, and that due to the accretion mode (disk structure), will have a different temporal signature (periodicity), which would make them easy to distinguish.

In this paper we show that changes in the orientation of the inner accretion disk of Cyg X-1 would affect the shape of the broad $\mathrm{Fe} \mathrm{K} \alpha$ emission line in a periodic way. Under the assumption that the X-ray spectrum of Cyg X-1 is not substantially more complex than what has already been found in the 
Chandra observation, eV-level spectral resolution observations of the system (as those that will be carried out by the Astro-E2 satellite) can be used to constrain the dynamics of the disk and establish whether the accretion disk of Cyg X-1 actually precesses and, if so, determine its dynamics.

\section{DISK PRECESSION IN CYGNUS X-1}

Several X-ray binaries present periodic behavior in their light curves on timescales longer than the orbital period. Among these systems we can mention Her X-1, SS 433, and LMC X-4. It has been suggested that these long periods correspond to the precession of the accretion disk (e.g., Katz 1973). In the case of SS 433 the precession is directly measured in the jets, so if these are attached to the accretion disk, it is reasonable to expect that the disk will also display precession (Katz 1980). Although there is no compelling evidence yet reported for disk precession in black hole binaries, it is reasonable that the same mechanisms responsible for this phenomenon in neutron binaries will apply.

The mechanism that produces the precession might be the instability of the response of the disk to the radiation reaction force from the illumination by the central source (e.g., Wijers \& Pringle 1999; Ogilvie \& Dubus 2001) or the tidal force of the companion star on a disk that is not coplanar with the binary orbit (Katz 1973; Larwood 1998; Kaufman Bernadó et al. 2002). Uniform disk precession will occur in this case only if the sound crossing time through the disk is considerably shorter than the characteristic precession period induced by the perturbing star. The precession angular velocity is given by $\left|\Omega_{p}\right| \approx$ $\frac{3}{4}\left(G m / r_{m}^{3}\right)\left(1 / \omega_{d}\right) \cos \theta$ (e.g., Romero et al. 2000), where $G$ is the gravitational constant, $r_{m}$ is the orbital radius, $\omega_{d}$ is the inner disk angular velocity, $\theta$ is the half-opening angle of the precession cone, and $m$ is the mass of the star that exerts the torque upon the disk. The orbital period $T_{m}$ is related with the involved masses and the size of the orbit by Kepler's law, $r_{m}^{3}=[G(m+$ $\left.M) T_{m}^{2}\right] / 4 \pi^{2}$, where $M$ is the mass of the accreting object. The ratio between the orbital and the precessing periods can be related through the disk angular velocity $\omega_{d}=\left(G M / r_{d}^{3}\right)^{1 / 2}$ :

$$
\frac{T_{m}}{T_{p}}=\frac{3}{4} \frac{m}{M} \kappa^{3 / 2}\left(\frac{M}{m+M}\right)^{1 / 2} \cos \theta,
$$

where $T_{p}=2 \pi / \Omega_{p}$ and $\kappa=r_{d} / r_{m}$. Since $\kappa<1$, normally $T_{m} / T_{p}<1$. In the case of Cyg X-1, Brocksopp et al. (1999) have reported multiwavelength evidence for the existence of a period of $142.0 \pm 7.1$ days. Similar precessing periods have been calculated by Larwood (1998), Katz (1973, 1980), and Katz et al. (1982) for other X-ray binaries on the basis of the same model. As shown by Romero et al. (2002) in the case of Cyg X-1, such a period can be obtained from tidally induced precession for an accretion disk with a size $\sim 4 \times 10^{11} \mathrm{~cm}$, if the half opening angle of the precession cone is $\sim 15^{\circ}$. For a purely wind-fed system, this size might be too large, and other mechanisms may be in operation to generate the observed timescales. In particular, radiation-driven precession (Pringle 1996; Maloney \& Begelman 1997; Ogilvie \& Dubus 2001), wind-driven warping and precession (Schandl \& Meyer 1994; Quillen 2001), and spin-spin precession (Bardeen \& Petterson 1975; Armitage \& Natarajan 1999) can yield precession periods of several weeks to a few months. In all these mechanisms the observed optical and X-ray modulation points to precession of the disk, whereas the radio variations might originate in the jet.

\section{Fe $\mathrm{K} \alpha$ LINE PROFILE DIAGNOSIS}

The use of emission lines as a diagnosis tool for the state of binary systems has been proposed in the past (e.g., for an investigation on supermassive black hole binarity; see Torres et al. 2003; also Gaskell 1983; Zakharov 2004; Zakharov et al. 2004). Here we show that a similar method can be used to extract information about the precession status of microquasars.

For the case of Cyg X-1, we assume that the time-averaged disk inclination angle is $35^{\circ}$, which is in agreement with the fitting of the system's Fe line (Miller et al. 2002). Values around this time-averaged inclination angle were also found for other binary systems ( e.g., Her X-1, LMC X-4, SMC X-1, etc., as discussed by Larwood 1998). We also assume two extreme cases for the amplitude of the disk precession: (1) the disk inclination angle precesses from $31^{\circ}$ to $39^{\circ}$ (very low magnitude of the precession angle) and (2) the disk inclination angle precesses from $5^{\circ}$ to $65^{\circ}$ (large magnitude of the precession angle). The amplitude of the precession of the inner disk should not be large if it is due to the tidal force of the secondary star and if the disk (especially the outer disk region) develops a significant warp. However, if the initial spin direction of the $\mathrm{BH}$ is significantly different from the orbital angular momentum direction, the inner disk, which is confined to the equatorial plane of the $\mathrm{BH}$ (if the spin is high) due to the Bardeen-Peterson effect, may precess around the total angular momentum (dominated by the orbital angular momentum) with an amplitude as large as the initial orbital inclination angle with respect to the $\mathrm{BH}$ equatorial plane. If we assume that the disk is rigidly precessing around the total angular momentum (dominated by the orbital angular momentum), then the amplitude of the precession may also be around $30^{\circ}$.

Several calculations on the disk line profiles have been performed. We use a ray-tracing technique and elliptic integrals (Rauch \& Blandford 1994; see also Yu \& Lu 2000; Lu \& Yu 2001 and references therein) to follow the trajectories of photons from the observer, keeping track of all coordinates until the photons either intersect the accretion disk plane, disappear below the event horizon, or escape to "infinity" (operationally defined to be $r=1000 \mathrm{GM} / \mathrm{c}^{2}$ away from the $\mathrm{BH}$ ). We then calculate the redshift factor for a photon (to the observer) emitted from a particular position on the disk. The solid angle subtended at the observer by each disk element is also calculated. We set the inner radius of the disk to be at the marginally stable orbit $\left(6 r_{g}\right.$ for a Schwarzschild black hole or $1.23 r_{g}$ for a Kerr black hole with spin $a / M=0.998$, where $r_{g}=G M / c^{2}$ ) and the outer radius at $160 r_{g}$. We assume that the surface emissivity of line photons follows a power law, $r^{-q}$, with $q=2.5$. Both the power-law emissivity law and the size of the disk in Schwarszchild units, are the usual assumptions (see, e.g., Nandra et al. 1997). The BH spin is assumed to be $a / M=0.998$. In microquasar systems, both the high-frequency quasi-periodic oscillation and relativistic lines suggest a high spin. In any case, we proved that if we were to assume a lower spin, there is not much qualitative difference for the problem we have studied here. With the above assumptions, we sum up all the photons received by the observer, which is emitted from each disk element, and obtain the profile of emergent $\mathrm{Fe} \mathrm{K} \alpha$ lines, with different inclination angles from the Cyg X-1 system, as shown in Figure 1.

\section{OBSERVING THE Fe LINE PROFILE VARIATIONS}

The complex X-ray spectrum of Cyg X-1 revealed by the Chandra observation reported in Miller et al. (2002) implies 

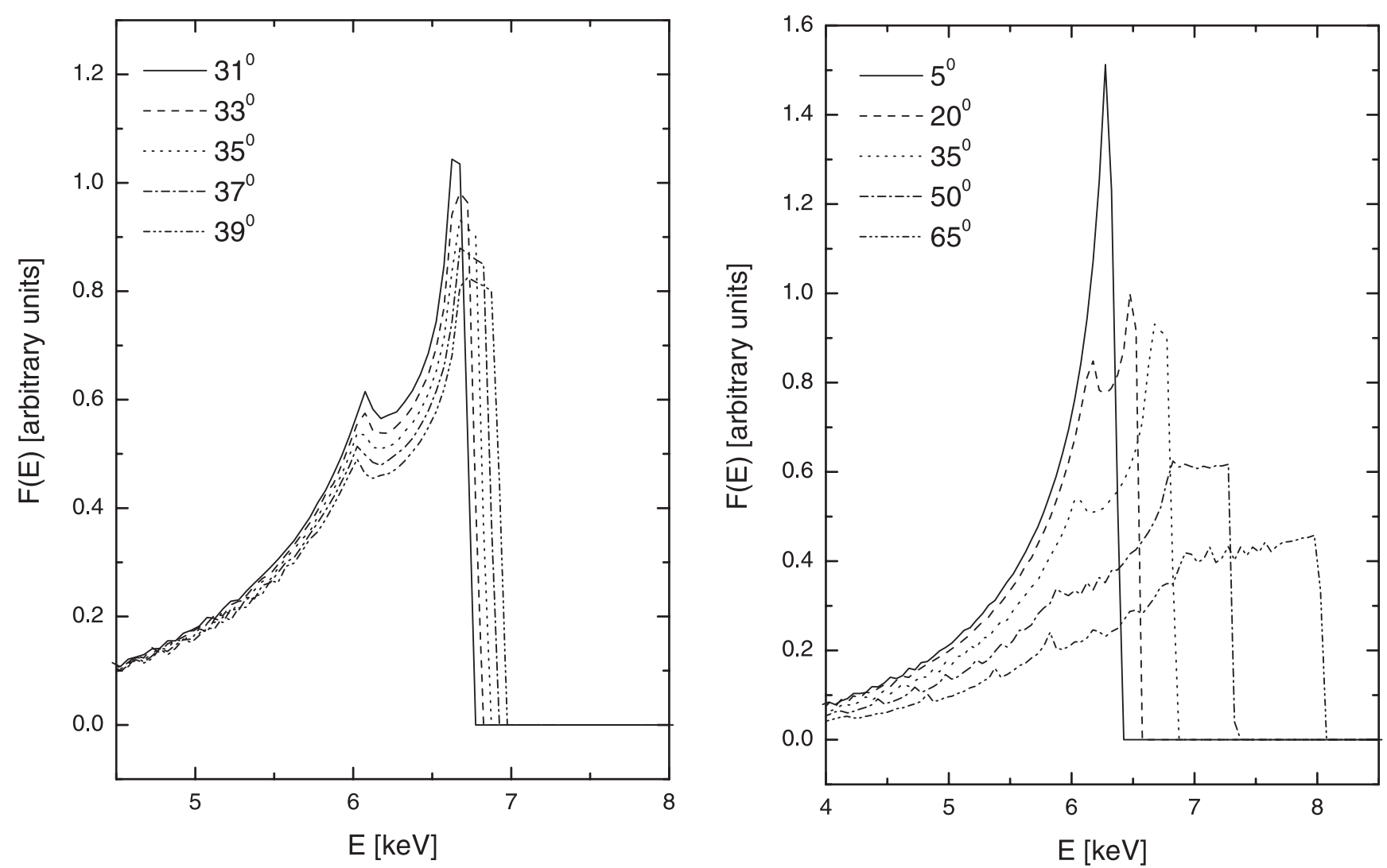

FIg. 1.-Shift in the Fe K $\alpha$ line profile of Cyg X-1 as a function of different inclination. The total line flux of each line is normalized to 1.

that the detection of minute variations in the Fe line shape will require a high spectral resolution instrument with large throughput. In what follows, we discuss the feasibility of detecting the precession of the accretion disk with Astro-E2, and specifically with the X-Ray Spectrometer (XRS) consisting of an array of semiconductor-based calorimeters delivering the best spectral resolution to date at $6 \mathrm{keV}$ (preflight value of $6.5 \mathrm{eV}$ ). Astro-E2 is a JAXA/NASA mission to observe X-rays with unprecedented high spectral resolution imaging detectors, which is scheduled for launch by the summer of 2005. The Astro-E2 Science Working Group target list includes two observations of Cyg X-1.

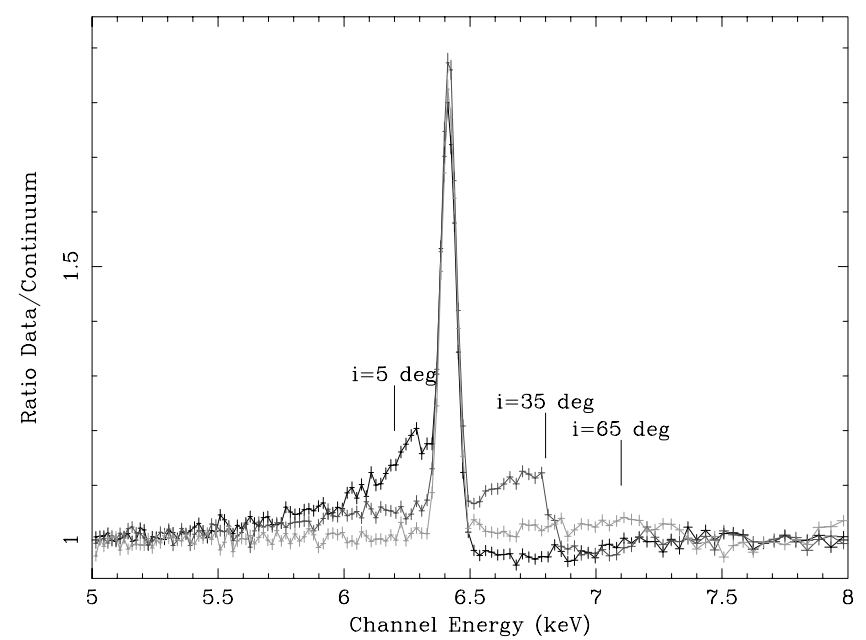

The underlying assumption is that the X-ray spectrum of Cyg X-1 can be well described by the best-fit model obtained by Miller et al. (2002). Indeed, at higher spectral resolution, otherso far undetected - spectral components might appear. Examples include the presence of blueshifted edges due to high velocity ejecta. Should these, or other, features impact strongly on either the Fe line region itself or the energy ranges in which the underlying continuum is estimated, the specific methodology proposed here will need to be revised. Fortunately, the instrument selected will deliver spectra with a very high signal-tonoise ratio at a spectral resolution so high that many of these putative components might be resolved and modeled out. That

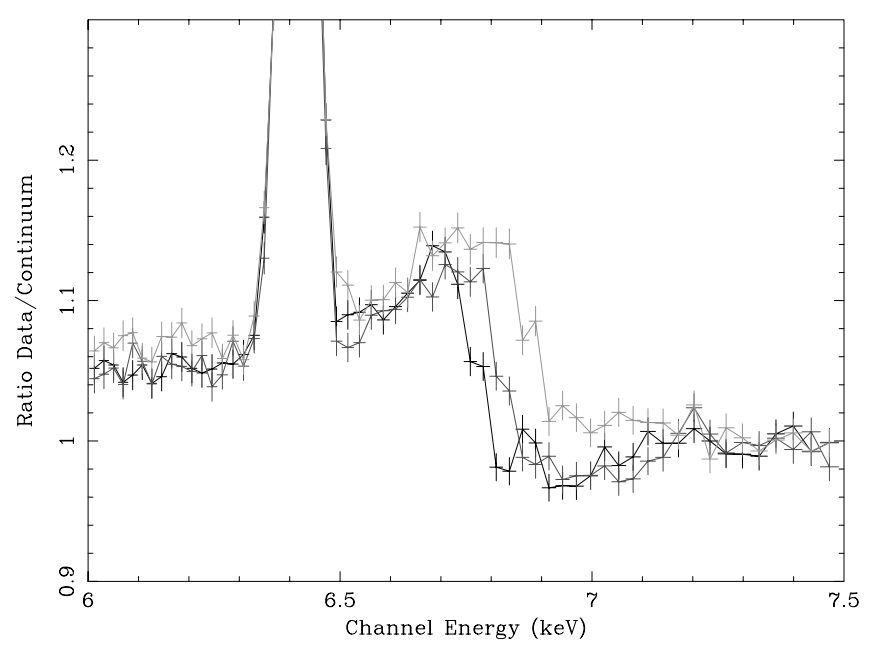

FIG. 2.-Left: Ratio of data to fitted continuum for net $50 \mathrm{ks}$ exposures, assuming inner disk inclination angles of $5^{\circ}, 35^{\circ}$, and $65^{\circ}$. Right: Detail of the ratio of data to

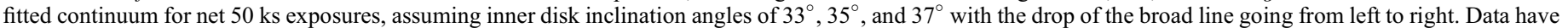
been grouped for fitting and presentation purposes. [See the electronic edition of the Journal for a color version of this figure.] 
TABLE 1

Disk Parameters, with 90\% Errors, for Various Effective Exposure Times

\begin{tabular}{|c|c|c|c|c|}
\hline Parameter & Input & $50 \mathrm{ks}$ & $10 \mathrm{ks}$ & $5 \mathrm{ks}$ \\
\hline . & 1.80 & $1.80 \pm 0.010$ & $1.80 \pm 0.015$ & $1.80 \pm 0.02$ \\
\hline$E_{\text {edge }}(\mathrm{keV}) \ldots \ldots \ldots \ldots \ldots \ldots$ & 7.20 & $7.19 \pm 0.05$ & $7.20 \pm 0.10$ & $7.12 \pm 0.15$ \\
\hline$E_{\text {narrow }}(\mathrm{keV}) \ldots \ldots \ldots \ldots \ldots$ & 6.415 & $6.414 \pm 0.001$ & $6.414 \pm 0.001$ & $6.416 \pm 0.002$ \\
\hline 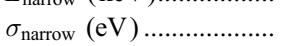 & 30 & $29.3 \pm 0.6$ & $29.3 \pm 1.2$ & $32.0 \pm 2.0$ \\
\hline Disk inclination............... & 35 & $35.0 \pm 0.3$ & $35.2 \pm 0.6$ & $35.3 \pm 0.7$ \\
\hline
\end{tabular}

would certainly complicate, but not invalidate, the proposed analysis.

As argued by Miller et al. (2002), the spectrum of Cyg X-1 is complex below $3 \mathrm{keV}$, where they did not succeed in fitting an appropriate model. All our discussion assumes the Be filter is on at the XRS. This removes low-energy photons that are not needed for our purposes. In our analysis, we ignore photons below $3 \mathrm{keV}$ and above $9 \mathrm{keV}$. In addition, we do not use the X-Ray Imaging Spectrometers (XIS) on board Astro-E2, which have less spectral resolution at the Fe line energy range. A typical $50 \mathrm{ks}$ exposure is assumed, and the background is assumed to be negligible for such a strong source (see below) and not included in the simulations. Prelaunch calibration redistribution matrices and efficiency curves have been downloaded from the Astro-E2 Web site at NASA. ${ }^{5}$

The simulated model is that fitted by Miller et al. (2002) to the Chandra data. The continuum is a power law with $\Gamma=1.8$, absorbed by a Galactic column of $N_{\mathrm{H}}=8.1 \times 10^{21} \mathrm{~cm}^{-2}$, which is absorbed by a smeared edge at $7.2 \mathrm{keV}$, with a width of $7 \mathrm{keV}$ and a depth of 1.2. The narrow-line component at $6.415 \mathrm{keV}$, believed to arise from the irradiated outer disk, has been simulated as a Gaussian of width $\sigma_{\text {narrow }}=30 \mathrm{eV}$. The broad-line component has been simulated using the numerical models explained in the previous section, for a fixed equivalent width of $140 \mathrm{eV}$ and a variety of disk inclination angles.

As expected, this model produces a very high count rate in the XRS, of the order of 60 counts s ${ }^{-1}$. This count rate will be distributed among several XRS pixels, according to the pointspread function. Although the overall count rate is below the telemetry limit, the fraction of events that will be measured by the on-board software at medium or low resolution will be large $(\sim 30 \%-40 \%)$. There are two possibilities to deal with this: either using the neutral density filter (which will decrease the overall count rate to an acceptable level of $\sim 6$ counts $\mathrm{s}^{-1}$ ) or ignoring the few pixels with the higher count rates and working only with the pixels that have count rates below a few counts $\mathrm{s}^{-1}$. Both of these procedures will result in an overall loss of throughput. This is why we consider effective exposures of 10 and $5 \mathrm{ks}$.

To analyze the simulated data, we follow Miller et al. (2002) to fit the continuum by excluding the range from 4.0 to $7.2 \mathrm{keV}$. A single power law leaves enormous residuals that can be well fitted by the smeared absorption edge. In general, the edge en-

\footnotetext{
${ }^{5}$ See http://astroe2.gsfc.nasa.gov/docs/astroe/prop_tools/xrs_mat.html, with the most recent updates included (as of 2004 August 4).
}

ergy is well reproduced by the fit (statistical $90 \%$ errors in the range of 50-150 eV depending on the effective exposure time), although there is substantial degeneracy between the width of the edge and its depth. This does not affect the continuum in the Fe emission-line region.

Once the continuum is fitted, we include all data in the 3.09.0 energy band and add a narrow Gaussian emission line and a relativistic emission line (see Fig. 2, left). Thanks to the superb spectral resolution of this instrument, the narrow line is very well characterized, with errors in its centroid actually limited by systematics $(2 \mathrm{eV})$ rather than by statistics, even in a $5 \mathrm{ks}$ exposure. The relativistic line model returns the disk inclination angle with a $90 \%$ error of 0.3 for a $50 \mathrm{ks}$ exposure and 0.7 for a $5 \mathrm{ks}$ exposure. This is due to the fact that the sharp drop in the blue edge of the line is very clearly marked by the XRS. Figure 2 (right panel) shows the differences in these sharp edges for a $50 \mathrm{ks}$ exposure and various disk inclination angles. Note that these changes amount to about $50 \mathrm{eV}$ per degree of disk inclination, and therefore the claimed limit in the systematics for line centering of $2 \mathrm{eV}$ is really not an issue for this purpose. Table 1 summarizes the results of the fits to simulated data with 5,10 , and $50 \mathrm{ks}$ net exposure and disk inclination of $35^{\circ}$.

\section{CONCLUDING REMARKS}

This work suggests a diagnostic tool to investigate whether the accretion disk of Cyg X-1 (and those of other microquasar systems) precesses, and if so, determine its period and precession angle. We have shown that the study of the periodic variations of the $\mathrm{Fe} \mathrm{K} \alpha$ line, which would be unavoidably produced in the putatively precessing disk of the system, are observable in short (5-10 ks) exposures of the Astro-E2 satellite. The degree of precision and confidence level up to which we are able to determine the inclination angle of disk for each short observations and, thus, the magnitude of the precession is shown to be sufficiently high as to allow a clear determination of these parameters even when the precession angle is of only a few degrees.

The work of D. F. T. was performed under the auspices of the US DOE-NNSA by the University of California Lawrence Livermore National Laboratory under contract W-7405-Eng48. G. E. R. is supported by the research grant PICT 03-13291 (ANPCT). X. B. acknowledges financial support by the Spanish Ministerio de Educación y Ciencia, under project ESP 200300852 .
Armitage, P. J., \& Natrarajan, P. 1999, ApJ, 525, 909

Bardeen, J. M., \& Petterson, J. A. 1975, ApJ, 195, L65

Barr, P., White, N. E., \& Page, C. G. 1985, MNRAS, 216, P65

Brocksopp, C., Fender, R. P., Larimov, V., Lyuty, V. M., Tarasov, A. E., Pooley, G. G., Paciesas, W. S., \& Roche, P. 1999, MNRAS, 309, 1063

\section{REFERENCES}

Dove, J. B., Wilms, J., Maisack, M., \& Begelman, M. G. 1997, ApJ, 487, 759 Ebisawa, K., et al. 1996, ApJ, 467, 419

Esin, A. A., McClintock, J. E., \& Narayan, R. 1997, ApJ, 489, 865

Esin, A. A., Narayan, R., Cui, W., Grove, J. E., \& Zhang, S. 1998, ApJ, 505, 854 
Fender, R. P. 2001, MNRAS, 322, 31

Fender, R. P., Belloni, T. M., \& Gallo, E. 2004, MNRAS, 355, 1105

Gaskell, C. M. 1983, in Proc. 24th Liège Int. Astrophys. Colloq., Quasars and Gravitational Lenses (Liège: Univ. Liège), 473

Gierliński, M., Zdziarski, A. A., Poutanen, J., Coppi, P. S., Ebisawa, K., \& Johnson, W. N. 1999, MNRAS, 309, 496

Herrero, A., Kudritzki, R. P., Gabler, R., Vilchez, J. M., \& Gabler, A. 1995, A\&A, 297, 556

Katz, J. I. 1973, Nature Phys. Sci., 246, 87 1980, ApJ, 236, L127

Katz, J. I., Anderson, S. F., Margon, B., \& Grandi, S. A. 1982, ApJ, 260, 780 Kaufman Bernadó, M. M., Romero, G. E., \& Mirabel, I. F. 2002, A\&A, 385, L10

Kitamoto, S., et al. 1990, PASJ, 36, 731

Larwood, J. D. 1998, MNRAS, 299, L32

Lu, Y., \& Yu, Q. 2001, ApJ, 561, 660

Maloney, P. R., \& Begelman, M. C. 1997, ApJ, 491, L43

Miller, J. M., et al. 2002, ApJ, 578, 348

Nandra, K., George, I. M., Mushotzky, R. F., Turner, T. J., \& Yaqoob, T. 1997, ApJ, 477, 602
Ogilvie, G. I., \& Dubus, G. 2001, MNRAS, 320, 485

Poutanen, J., Krolik, J. H., \& Ryde, F. 1997, MNRAS, 292, L21

Pringle, J. E. 1996, MNRAS, 281, 357

Quillen, A. C. 2001, ApJ, 563, 313

Rauch, K., \& Blandford, R. D. 1994, ApJ, 421, 46

Reynolds, C. S., \& Nowak, M. A. 2003, Phys. Rep., 377, 389

Romero, G. E., Chajet, L., Abraham, Z., \& Fan, J. H. 2000, A\&A, 360, 57

Romero, G. E., Kaufman Bernadó, M. M., \& Mirabel, I. F. 2002, A\&A, 393, L61

Schandl, S., \& Meyer, F. 1994, A\&A, 289, 149

Stirling, A. M., Spencer, R. E., de la Force, C. J., Gartett, M. A., Fender, R. P., \& Ogley, R. N. 2001, MNRAS, 327, 1273

Tanaka, Y. 1991, Fe Line Diagnostics in X-Ray Sources, ed. A. Treves (Berlin: Springer), 98

Torres, D. F., Romero, G. E., Barcons, X., \& Lu, Y. J. 2003, ApJ, 596, L31

Wijers, R. A. M. J., \& Pringle, J. E. 1999, MNRAS, 308, 207

Yu, Q., \& Lu, Y. 2000, MNRAS, 311, 161

Zakharov, A. F. 2004, preprint (astro-ph/0411611)

Zakharov, A. F., Ma, Z., \& Bao, Y. 2004, NewA, 9, 663 